\title{
Thoracic outlet syndrome- A myofascial variant: Part 2. Treatment
}

\author{
BENJAMIN MARC SUCHER, DO
}

Thoracic outlet syndrome (TOS) may be treated successfully with a powerful form of myofascial release manipulation and stretching. Self-stretching exercises complement all other treatment modalities for TOS. Maximal effect is achieved with high-frequency, progressive stretching, tapered rapidly to a maintenance level as symptoms diminish. Stretching must be demonstrated "hands on" with the patient and reviewed and modified regularly. The techniques are similar to those of Travell and Simons, with modifications. The pain of TOS is intimately connected with short, contracted muscles that develop trigger points. An engram for the shortened muscles develops centrally. The myofascial release technique involves local release of myofascial structures, re-energizing of the tissues, and reprogramming of the central engram for the particular muscle length.

Part 1 described four cases of thoracic outlet syndrome (TOS) with use of thermography as a diagnostic aid. ${ }^{1} \mathrm{~A}$ form of deep myofascial release combined with self-stretching exercise was briefly mentioned as a rapidly effective treatment method.

Alternative forms of treatment were sought because intensive courses of physical therapy along with various medications have been relatively unrewarding in many cases. Various forms of manipulation, such as muscle energy and functional release techniques, at times appeared promising, yet fell short of producing ideal results. The classic TOS exercises ${ }^{2}$ have been used with mixed results, often aggravat-

Reprint requests to Benjamin Marc Sucher, DO, 10555 N Tatum Blvd, Suite A-104, Mountain View Center, Paradise Valley, AZ 85253. ing symptoms or not leading to any improvement. These frustrations led to the blending of various forms of treatment to produce a more dynamic alternative.

Others ${ }^{3,4}$ have mentioned the use of osteopathic myofascial treatment techniques for TOS but provided no specific descriptions. This article describes the treatment method in detail, reviews theoretical bases for its effectiveness, and illustrates some of the self-stretching techniques.

\section{Procedure}

The protocol of treatment for most mild to moderate cases of TOS is relatively simple: decrease the pain, relax the muscles, stretch and release the myofascia, and correct posture. The process, however, is somewhat difficult. The protocol is as follows:

1. Analgesic, anti-inflammatory medications to take the "edge off" the pain.

2. Muscle relaxants (cyclobenzaprine hydrochloride works best), especially at bedtime, to facilitate sleep.

3. Physical therapy modalities such as superficial heat, ultrasound, or electrical muscle stimulation. These are optional but most helpful with more chronic and severe cases. Such modalities help decrease pain. The ultrasound renders the myofascia more elastic and stretchable, but patients must stretch or be treated with manipulation (or both) within 30 minutes after treatment.

4. Vigorous, progressive stretching exercises (five to ten times daily).

5. Manipulative treatment consisting of local myofascial release to restricted, contracted muscles or fascial structures (or both), to improve postural alignment, and to achieve structural symmetry.

6. Postural awareness and correction, heel lift (as appropriate), and reduction or elimina- 

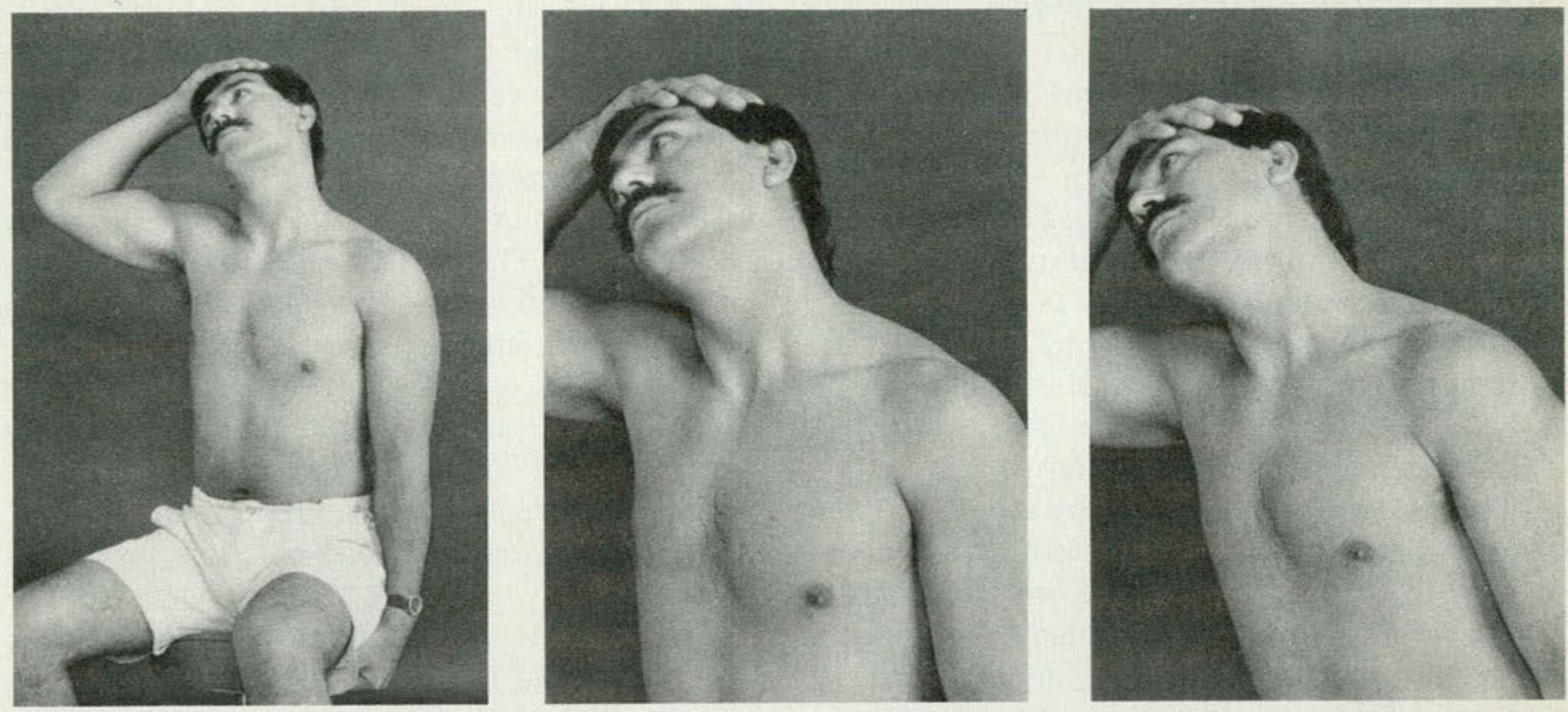

Figure 1. Stretching technique for the scalene (anterior and middle) muscle. Left: The arm on the side to be stretched is secured down (hooked under seat) to allow more control and effective stretch. The opposite hand wraps partially around the head for good control to assist with the stretch (center). After proceeding as far as tolerated, the patient leans the whole trunk away from the side being stretched, creating additional traction (downward) on the muscle by the arm that is secured (right).
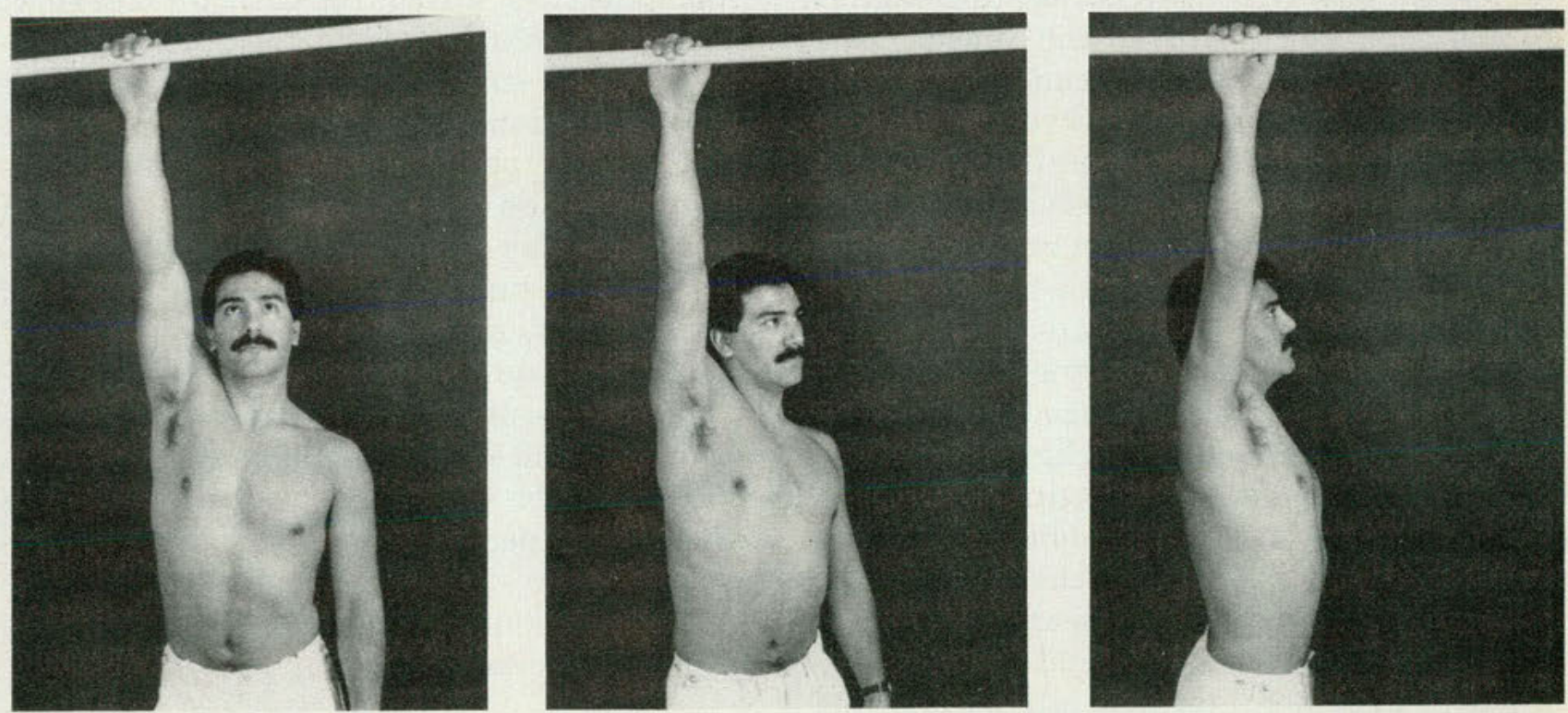

Figure 2. Stretching technique for the smaller pectoral muscle. Left: The patient has taken the slack out of the muscle. He then rotates the body away from the side being stretched, increasing traction (center). Maximum rotation and stretch effect are achieved slowly (right). 
tion of occupational and mechanical stressors (eg, lifting and repetitive tasks).

\section{Self-stretching exercise technique}

The techniques used are basically similar to those described by Travell and Simons, ${ }^{5}$ with modifications to include self-application of positioning leverage. An additional "multiplier" is used for the seated techniques (Fig 1), which creates a counterforce to enhance the stretch effect. Another modification is used for the smaller pectoral muscle, which is stretched when the patient hangs by the arm from an overhead source, such as bar, stairs, or door frame, as demonstrated in Figure 2.

The patient is instructed initially to create some tension or pulling sensation in the involved muscle, which should be felt as some localized discomfort. This tension is held for 10 to 30 seconds while slow, deep breathing is begun. After exhaling, the patient is encouraged to "take up the slack" that has developed, increasing pull in small, successive increments until no additional amount can be achieved or tolerated. This procedure is repeated five to ten times throughout the day.

\section{Myofascial release technique}

The technique involves stretching the particular muscle involved, with positioning similar to that described by Travell and Simons. ${ }^{5}$ The work on the scalene muscles can be performed with the patient seated; however, it is somewhat more effective and easier on the patient if he or she is supine or side-lying. The sidelying position, with no support under the head, places the scalene muscles on the "up side," under stretch. With the operator standing behind the patient and supporting or retracting (downward-inferior pull) the shoulder with one hand, good control can be achieved with the other hand in manually releasing the scalene muscles (Fig 3 left). Some additional release can usually be obtained by then having the patient roll supine, with the operator laterally flexing the neck with one hand while releasing with the other. Placing a pillow under the patient's thorax or extending the patient's head off the table allows increased cervical extension which, combined with sidebending, will increase stretch on the anterior scalene muscle (Figs 3 right, top and right, bottom). The release is effected by using the tips or sides (or both) of the second and third digits, starting high, near the origin of the muscle in the mid to upper cervical area and slowly moving inferiorly toward the insertion as the neck and head are flexed laterally.

Slow, deep breathing aids patient relaxation and release. The patient breathes into the operator's hand while the operator maintains firm contact with the muscle. Then, during or after exhalation, the operator can advance the hand deeper into or along the muscle, taking up the slack. The operator continues advancing as far as possible, sensing release, and adjusting pressure and head position while proceeding.

The smaller pectoral muscle can also be treated while the patient is supine or sidelying. With the patient side-lying, the operator stands behind the chest and places one hand laterally beneath the greater pectoral muscle to contact the smaller pectoral muscle. The other hand supports the shoulder joint anterolaterally and retracts posteriorly. Firm pressure is simultaneously applied to the smaller pectoral muscle, starting superiorly near the coracoid process and slowly moving inferiorly along the chest wall as the patient takes slow, deep breaths (Fig 4 right, top). With the patient in the supine position, traction on the abducted extended arm facilitates treatment (Fig 4 right, top). Alternatively, vigorous passive retraction of the scapula can aid stretch and release by applying firm downward pressure on the humeral head with the palm of the operator's one hand, while the other hand applies pressure on the muscle. A pillow underneath the mid thorax produces some spine extension and aids in shoulder retraction. The muscle can be approached ventrally, through the greater pectoral muscle, or laterally under the more superficial pectoral muscle, the latter technique usually being more effective (Fig 4 right, bottom).

\section{Discussion}

Management in the cases described in Part $1^{1}$ focused on a form of osteopathic manipulation

(continued on page 817) 


\section{HYPERTENSION IS USUALLY SILENT}

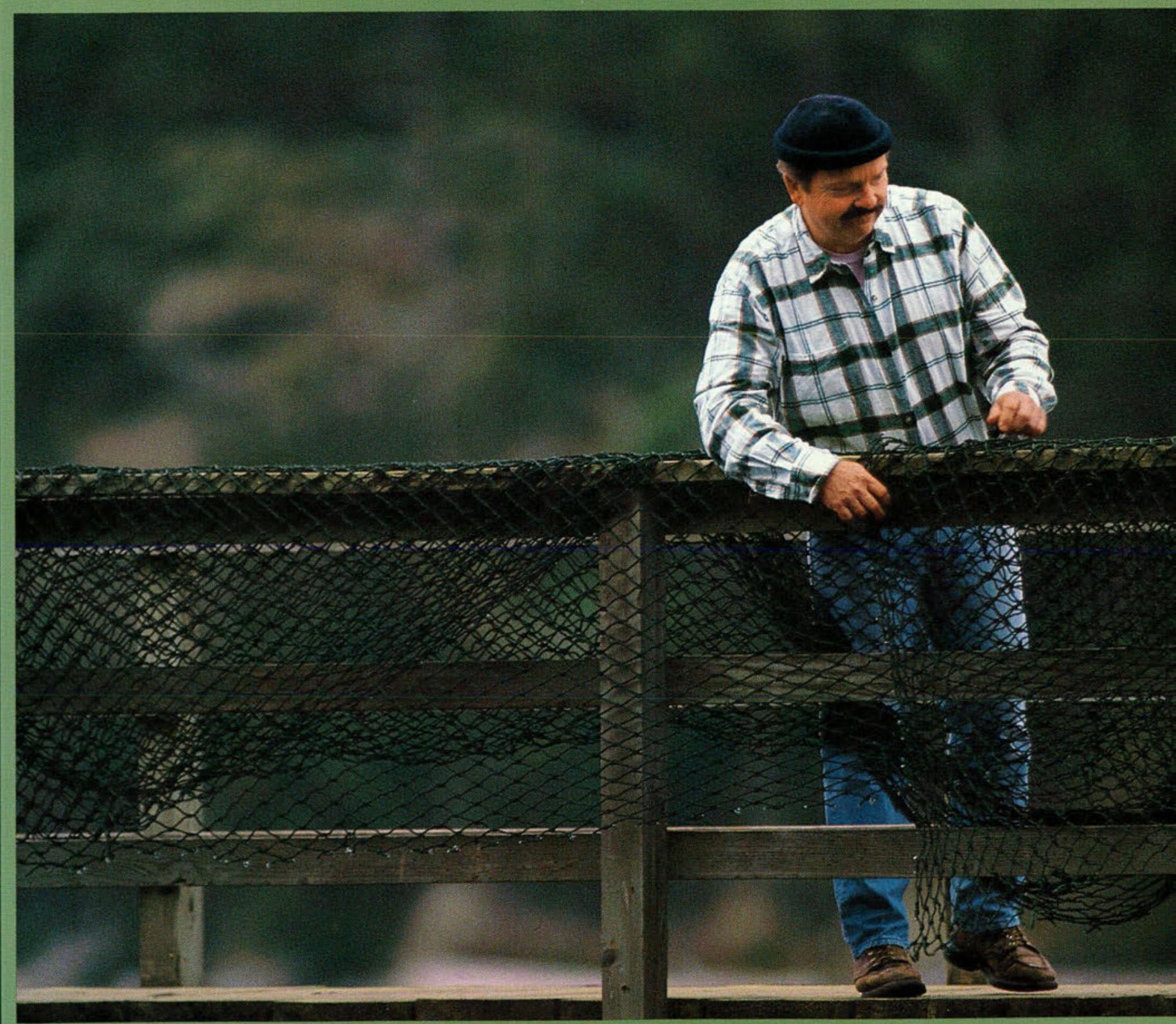




\section{THERAPY THAT MAY BE AS SILENT AS HYPERTENSION ITSELF}

VASOTEC ${ }^{\circledR}$ (Enalapril Maleate, MSD) is generally well tolerated and not characterized by certain undesirable effects associated with selected agents in other antihypertensive classes.

VASOTEC is contraindicated in patients who are hypersensitive to this product and in patients with a history of angioedema related to previous treatment with an ACE inhibitor.

A diminished antihypertensive effect toward the end of the dosing interval can occur in some patients.

For a Brief Summary of Prescribing information, please see the last page of this advertisement.

Copyright @ 1989 by Merck \& Co., INC. 

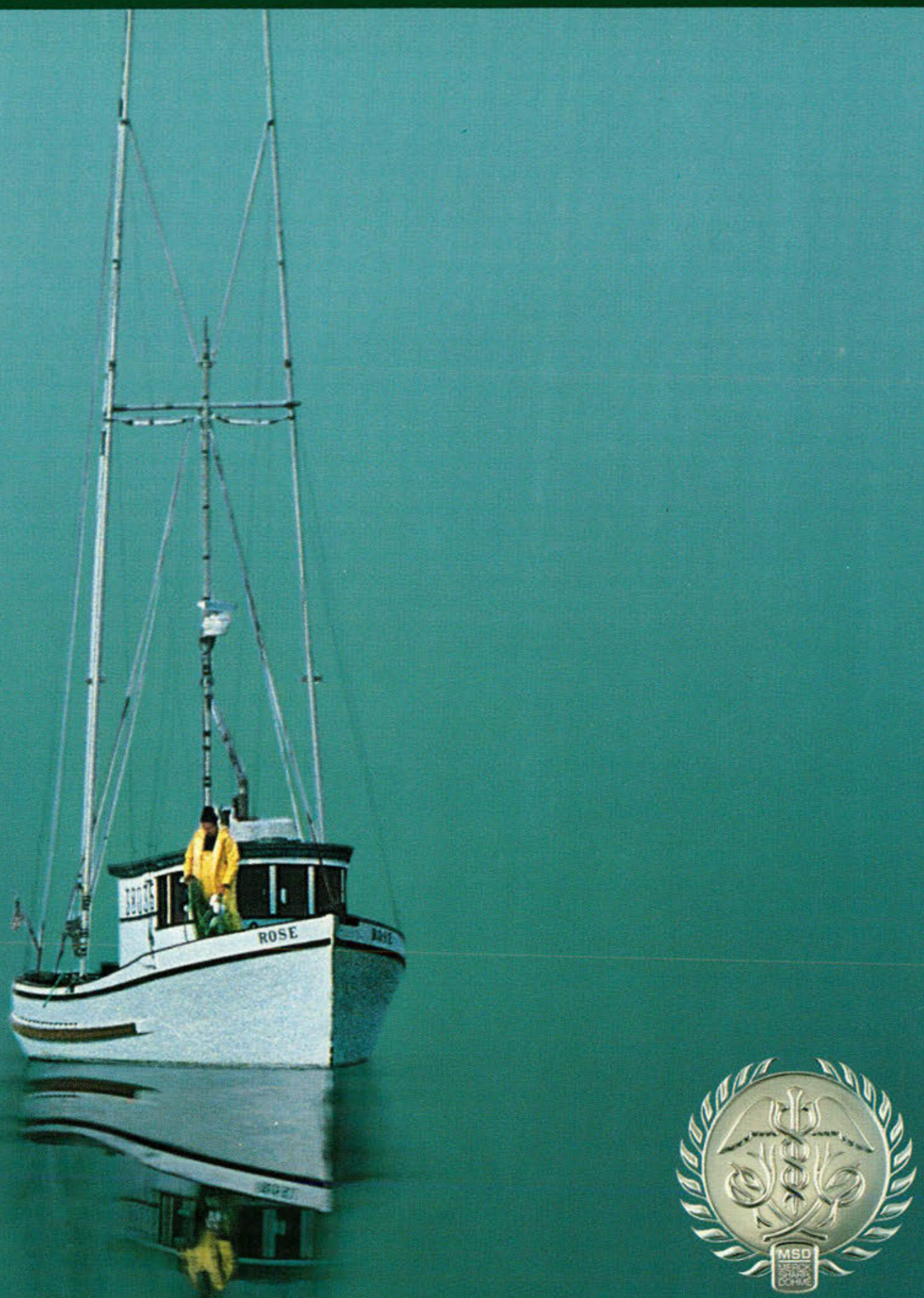

FOR MANY HYPERTENSIVE PATIENTS

ONCE-A-DAY VASOTEC

(ENALAPRIL MALEATE MSD) 


\section{NASOTIEC}

VASOTEC is available in 2.5-mg, 5-mg, 10-mg, and 20-mg tablet strengths.

Contraindications: VASOTEC (Enalapril Maleate, MSD) is contraindicated in patients who are hypersensitive

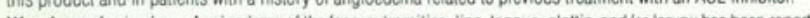
Warnings: Angioedema: Angioedema of the face, extremities, lips, tongue, glottis, and/or larynx has been reported in
patients ltreated with ACE inhibitors, including VASOTEC. In such cases, VASOTEC should be promptly discontinued and the patient carefully observed until the swelling disappears. In instances where swelling has been confined to the
tace and lips, the condition has generally resolved without treatment, although antihistamines have been useful in relieving symptoms. Angioedema associated with laryngeal edema may be fatal. Where there is involvement of the tongue, glottis, or larynx likely to cause airway obstruction, appropriate therapy, e.g. subcutaneous
epinephrine solution $1: 1000(0.3 \mathrm{~mL}$ to $0.5 \mathrm{~mL}$ ), should be promptly administered.' (See ADVERSE

Hypotension: Excessive hypotension is rare in uncomplicated hypertensive patients treated with VASOTEC alone Patients with heart failure given VASOTEC commonly have some reduction in blood pressure, especially with the first
dose, but discontinuation of therapy for continuing symptomatic bypotension usually is not necessary when dosing
instructions are followed; caution should be observed when initiating therapy. (See DOSAGE AND ADMINISTRA-
TION ) Patients at risk for excessive hypotension, sometimes associaled with oliguria and/or progressive azotemia

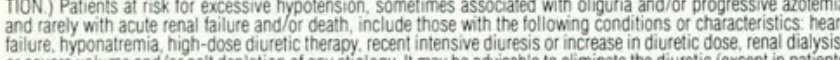
or severe volume and/or salt depletion of any etiology. It may be advisable to eliminate the diuretic (except in patients
with heart tailure), reduce the diuretic dose, or increase salt intake cautiously before initiating therapy with VASOTEC in patients at risk tor excessive hypotension who are able to tolerate such adjustments. (See PRECAUTIONS, Drug interactions and ADVERSE REACTIONS.) In patients at risk for excessive hypotension, therapy should be started unde
very close medical supervision and such patients should be followed closely for the first two weeks of treatment and whenever the dose of enalapril and/or diuretic is increased. Similar considerations may apply to patients with ischInfarction or cerebrovascular accident. If excessive hypotension occurs, the patient should be placed in the supin

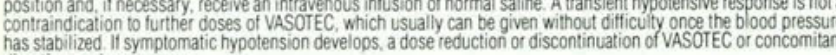
has stabilized. If symptomatic

Neutropenia/Agranulocytosis: Another ACE inhibitor, captopril, has been shown to cause agranulocytosis and bone marrow depression, rarely in uncomplicated patients but more trequently in patients with renal impairment, especially
it they also have a collagen vascular disease. Available data trom clinical trials of enalapril are insufficient to show that enalapril does not cause agranulocylosis at similar rates. Foreign marketing experience has revealed several cases of neutropenia or agranulocylosis in which a causal relationship to enalapril cannot be excluded Periodic mon car
white blood cell counts in patients with collagen vascular disease and renal disease should be considered

Precautions: General: Impaired Renal Function: As a consequence of inhibiting the renin-angiotensin-aldosterone system, changes in renal function may be anticipated in susceptible individuals. In patients with severe heart failure inhibitors, including VASOTEC. may be associated with oliguria and/or progressive azotemia and rarely with acute In clinical studies in hypertensive patients with unilateral or bilateral renal artery stenosis, increases in blood urea nitrogen and serum creatinine were observed in $20 \%$ of patients. These increases were almost aiways reversible upor
discontinuation of enalapril and/or diuretic therapy. In such patients, renal function should be monitored during the first few weeks of therapy

Some patients with hypertension or heart failure with no apparent preexisting renal vascular disease have developed ncreases in blood urea and serum creatinine, usually minor and transient, especially when VASOTEC has been given eduction and/or discontinuation of the diuretic and/or VASOTEC may be required

Evaluation of patients with hypertension or heart failure should always include assessment of renal

Hyperkalemia: Elevated serum potassium ( $>57 \mathrm{mE} / \mathrm{L}$ ) was observed in approximately $1 \%$ of hypertensive patients n clinical trials. In most cases these were isolated values which resolved despite continued therapy. Hyperkalem was a cause of discontinuation of therapy in $0.28 \%$ of hypertensive patients. In clin

Risk factors for the development of hyperkalemia include renal insufficiency, diabetes mellitus, and the concomitant use of potassium-sparing diuretics, potassium supplements, and/or potasi
should be used cautiously. if at all, with VASOTEC. (See Drug interactions.)

Surgery/Anesthesia In patients undergoing major surgery or during anesthesia with agents that produce hypotension considered to be due to this mechanism, it can be corrected by volume expansion.

Intormation for Patients

Angioedema: Angioedema, including laryngeal edema may occur especially following the first dose of enalapr Patients should be so advised and told to report immediately any signs or symptoms suggesting angioedema (swell-
ing of face, extremities. eyes, lips, tongue, difficulty in swallowing or breathing) and to lake no more drug until they ave consurted with the prescribing physician Hypotension: Patients should be cautioned to report lightheadedness, especially during the first few days of therapy
actual syncope occurs, the patients should be told to discontinue the drug until they have consulted with the prescrib-
ing physician. ing physician.

All patients should be cautioned that excessive perspiration and dehydration may lead to an excessive fall in blood pressure because of reduction in fluid volume. Other causes of volume depletion such as vom

Hyperkalemia: Patients should be told not to use salt substitutes containing potassium without consulting their physician.

Neutropenia: Patients showld

NOTE: As with many other drugs, certain advice to patients being treated with enalapril is warranted. This information intended effects

Drug interactions

Hypotension: Patients on Diuretic Therapy: Patients on diuretics and especially those in whom diuretic therapy was recently instituted may occasionally experience an excessive reduction of blood pressure after initiation of therapy diuretic or increasing the salt intake prior to initiation of treatment with enalapril. If it is necessary to continue the
diuretic, provide close medical supervision after the initial dose tor at least two hours and until blood pressure has

Agents Causing Renin Release: The antihypertensive effect of VASOTEC is augmented by antihypertensive agents tha Other Cardiovascular Agents: VASOTEC has been used concomitantly with beta-adrenergic-blocking agents, methyl-
dopa, nitrates, calcium-blocking agents, hydralazine, prazosin, and digoxin without evidence of clinically significan diverse interactions

Agents Increasing Serum Potassium: VASOTEC attenuates potassium loss caused by thiazide-type diuretics potassium-sparing diuretics (e.g. spironolactone, triamterene, or amiloride), polassium supplements, lant use of these agents is indicated because of demonstrated hypokalemia, they should be used with caution and
with frequent monitoring of serum potassium. Potassium-sparing agents should generally not be used in patients Lithium: Lithium toxicity has been reported in patients receiving lithium concomitantly with drugs which cause elim ination of sodium, including ACE infibitors. A few cases of lithium toxicity have been reported in patients receiving concomitant VASOTEC and lithium and were reversible upon discontinuation of both drugs it is recom
serum lithium levels be monitored trequently if enalapril is administered concomitantly with lithium

Pregnancy-Category C. There was no fetotoxicity or teratogenicity in rats treated with up to $200 \mathrm{mg} / \mathrm{kg} /$ day of enalapr

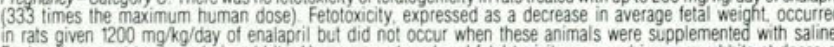
Enalapril was not teratogenic in rabbits. However, maternal and fetal toxicity occurred in some rabbits at doses of $\mathrm{kg} /$ day but not at $30 \mathrm{mg} / \mathrm{kg} / \mathrm{day}$ ( 50 times the maximum human dose)

Radioactivity was found to cross the placenta following administration of labeled enalapril to pregnant hamsters.

There are no adequate and well-controlled studies of enalapril in pregnant women. However, data are available that
show enalapril crosses the human placenta. Because the risk of fetal toxicity with the use of ACE inhibitors has no been clearly defined, VASOTECs (Enalapril Maleate, MSD) should be used during pregnancy only if the potential ben-

Postmarketing experience with all ACE inhibitors thus far suggests the following with regard to pregnancy outcome Inadvertent exposure limited to the first trimester of pregnancy has not been reported to afiect fetal oulcome adversely.
Fetal exposure during the second and third trimesters of pregnancy has been associated with fetal and neonatal morhidity and mortality.

When ACE inhibitors are used during the later stages of pregnancy, there have been reports of hypotension and decreased renal perfusion in the newborn. Oligohydramnios in the mother has also been reported, presumably repre senting decreased renal function in the fetus. Intants exposed in utero to ACE inhibitors should be closely observed pressure and renal perfusion with the administration of fluids and pressors as appropriate. Problems associated with prematurity such as patent ductus arteriosus have occurred in association with maternal use of ACE inhibitors, but is not clear whether they are related to ACE inhibition, maternal hypertension, or the underlying prematurity. Nursing Mothers: Milk in lactating rats contains radioactivity following administration of "C enalapril maleate. It is not known whether this drug is secreted in human milk. Because many drugs are secreted in human milk, caution should exercised when VASOTEC is given to a nursing mother.

Pediatric Use: Safety and effectiveness in children have not been established.

Adverse Reactions: VASOTEC has been evaluated for safety in more than 10,000 patients, including over 1000 patients treated for one year
trials involving 2987 patients

(4.3\%), and tatigue ( $3 \%$.

Other adverse experiences occurring in greater than $1 \%$ of patients treated with VASOTEC in controlled clinical trials HEARI FAILUPE: The most trequent clinical adverse experiences in both controlled and uncontrolled trials were: dizzi-
ness $(7.9 \%)$. hivpotension $(67 \%)$, orthostatic effects $(2.2 \%)$, syncope $(2.2 \%)$, cough $(2.2 \%)$, chest pain $(2.1 \%)$, and (2.1\%)

Other adverse experiences occurring in greater than $1 \%$ of patients treated with VASOTEC in both controlled and

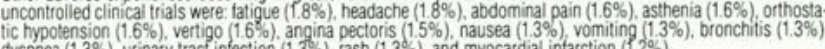

Other serious clinical adverse experiences occurring since the drug was marketed or adverse experiences occurring
in $0.5 \%$ to $1 \%$ of patients with hypertension or heart lailure in clinical trials in order of decreasing severity within each category.

Cardiovascular: Cardiac arrest; myocardial infarction or cerebrovascular accident, possibly secondary to excessive hypotension in high-risk patients (see WARNINGS, Hypd
edema: thythm disturbances, atrial fibrillation; palpitation.

Digestive: lleus, pancreatitis, hepatitis (hepatocellular or cholestatic jaundice), melena, anorexia, dyspepsia, constipation, glossitis. stomatitis, dry mouth

Muscuiloskeletal: Muscle cramps.

Nervous/Psychiatric: Depression, confusion, ataxia, somnolence, insomnia. nervousness, paresthesia

Urogenital: Renal tailure, oliguria, renal dystunction (see PRECAUTIONS and DOSAGE AND ADMINISTRATION) Respiratory: Bronchospasm, minorrhea, sore throat and hoarseness, asthma, upper respiratory infection. Skin: Exfoliative dermatitis, toxic epidermal necrolvsis. Stevens-Johnson syndrome, herpes zoster, erythema mult:.

Senses: Blurred vision, taste alteration, anosmia, tinnitus, conjunctivitis, dry eyes, tearing A symptom complex has been reported which may include a positive ANA, an elevated erythrocyte sedimentation rate, arthralgias/arthritis, myalgias, lever, serositis, vasculitis, leukocylosis, eosinophilia, photosensitivity. rash, and other

Angioedena: Angioederna has been reported in patients receiving VASOTEC $(0.2 \%)$. Angioedema associated with

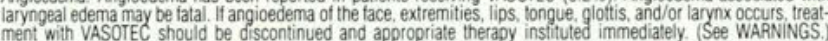
Hypotension: In the hypertensive patients, hypotension occurred in $0.9 \%$ and syncope occurred in $0.5 \%$ of patients following the initial dose or during extended therapy. Hypotension or syncope was a cause lor discontinuation of ther$2.2 \%$ of patients. Hypotension or syncope was a cause for discontinuation of therapy in $1.9 \%$ of patients with heart Clinical Laboratory Test Findings

Serum Electrolytes: Hyperkalemia (see PRECAUTIONS), hyponatremia.

Creatinine, Blood Urea Nitrogen: In controlled clinical trials, minor increases in blood urea nitrogen and serum cresion treated with VASOTEC a a lone Increases ara morere observed in about $0.2 \%$ of patients with essential hypertenpatients with renal artery stenosis. (See PRECAUTIONS, In patients with heart failure who were also receiving diuretics with or without digitalis, increases in blood urea nitrogen or serum creatinine, usually reversible upon discontinuation of VASOTEC and/or other concomitant diuretic therapy, were observed in about $11 \%$
Increases in blood urea nitrogen or creatinine were a cause for discontinuation in $1.2 \%$ of patients.

Hemoglobin and Hematocit: Small decreases in hemoglobin and hematocrit (mean decreases of approximately $0.3 \mathrm{~g} \%$ and 1.0 vol \%, respectively) Occur frequently in either hypertension or heart failure patients freated with
VASOTEC but are rarely of clinical importance unless another cause of anemia coexists. In clinical trials, less than

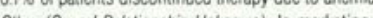

Other (Causal Relationship Unknown): In marketing experience, rare cases of neutropenia, thrombocytopenia, and bone marrow

Liver Function Tests: Elevations of liver enzymes and/or serum bilinubin have occurred.

Dosage and Administration: Hypertension: In patients who are currently being treated with a diuretic, symptomatic hypotension occasionally may occur following the initial dose of VASOTEC. The diuretic should, if possible, be discontinued for two to three days belore beginning therapy with VASOTEC to reduce the likelihood of hypotension. (See
WARNINGS) if the patient's blood pressure is not controlled with VASOTEC alone, diuretic therapy may be resumed. If the diuretic cannot be discontinued, an initial dose of $2.5 \mathrm{mg}$ should be used under medical supervision for at leas Wo hours and until blood
TIONS, Orug interactions.)

The recommended initial dose in patients not on diuretics is $5 \mathrm{mg}$ once a day. Dosage should be adjusted according divided doses. In some patients usual dosage range is 10 to $40 \mathrm{mg}$ per day administered in a single dose or in tw dosing interval. In such patients, an increase in dosage or twice-daily administration should be considered. If blood pressure is not controlled with VASOTEC alone, a diuretic may be added

Concomitant administration of VASOTEC with potassium supplements, potassitum
sparing duretics may lead to increases of serum polassium (see PRECAUTIONS).

Dosage Adjustment in Hypertensive Patients with Renal Impairment: The usual dose of enalapril is recommended tor patients with a creatinine clearance $>30 \mathrm{~mL} / \mathrm{min}$ (serum creatinine of up to approximately $3 \mathrm{mg} / \mathrm{dL}$ ). For patients may be titrated upward until blood pressure is controlled or 10 a maximum of $40 \mathrm{mg}$ daily.

Heart Failure: VASOTEC is indicated as adjunctive therapy with diuretics and digitalis. The recommended starting dose is $2.5 \mathrm{mg}$ once or twice daily. After the initial dose of VASOTEC, the patient should be observed under medica INGS and PRECAUTIONS, Drug Interactions), If possibile, the dose of the diuretic should be reduced, which may preclude subsequent careful dose titration with the drug following effective management of the hypotension. The usual therapeutic dosing range for the treatment of hearf tailure is 5 to $20 \mathrm{mg}$ daily given in two divided doses. The

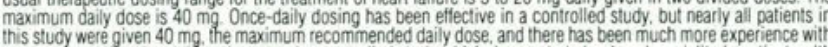
twice-daily dosing. In addifion, in a placebo-controlled study which, demonstrated reduced mortality in patients with severe heart failure (NYHA Class IN), patients were treated with 2.5 to $40 \mathrm{mg}$ per day of VASOTEC, almost always
administered in two divided doses. (See CLINICAL PHARMACOLOGY, Pharmacodnamics and Clinical Eflects.) Dosage administered in two divided doses. (See CLINICAL PHARMACOLOGY, Pharmacodnamics and
may be adjusted depending upon clinical or hemodynamic response. (See WARNINGS.)

Dosage Adjustment in Patients with Heart Failure and Renal Impairment or Hyponatremia: In patients with heart failure
who have hyponatremia (serum sodium $<130 \mathrm{mEg} / \mathrm{L}$ ) or with serum creatinine $>1.6 \mathrm{mg} / \mathrm{d}$. therapy should be initiated at $2.5 \mathrm{~m}$ d daily under close medical supervision. (See DOSAGE AND ADMINISTRATION. Hear? Failure, WARRINGS, and PRECAUTIONS, Drug interactions) The dose may be increased to $2.5 \mathrm{~m}$ bi.d. then $5 \mathrm{mg}$ b. id. and higher as needed, usually at intervals of four days or more, if at the time of dosage adjustment there is not excessive
tion. The maximum daily dose is $40 \mathrm{mg}$ For more detailed intormation, consult your MSO Representative or see Prescribing information, Merck
Sharp \& Dohme, Division of Merck \& Co. INC., West Point, PA 19486. J9VS5OR4(819) MSD
MERCK DOHME 
involving myofascial release and on progressive stretching exercises. This is considered to be an aggressive conservative approach, effective in treating myofascial pain syndromes, of which TOS is considered to be a variant. TOS is thought to be the ultimate challenge as a myofascial pain syndrome because it involves irritation of the neurovascular structures (by restricted, contracted myofascia) and postural or structural changes, all of which further irritate and stress the myofascia, creating a selfperpetuating vicious cycle. Properly executed, progressive stretching exercises complement the myofascial release treatment, synergistically helping to reverse the vicious cycle and progressively decrease myofascial tension, allowing posture to return to a closer approximation of normal and structure to become more symmetric.

\section{Stretching}

Probably the most important aspect of treatment is stretching. Some cases of TOS will resolve with good stretching technique alone. However, the type and angle of stretch and degree of pull are critical. Most patients require several sessions of exacting instruction. Operator-assisted stretch, often with vapocoolant spray and myofascial release manipulation, will get the patient started, allowing him or her to experience the direction of pull and intensity. Dichlorodifluoromethane works well with mild cases, but ethylchloride is necessary with more resistant ones.

It is important for patients to understand that initial treatment usually increases symptoms temporarily. This is simply because the process of stretching literally pulls already tight, shortened myofascial structures against the neurovascular bundle. In addition, the muscles themselves are sensitive and can be a direct source of pain when stretched. ${ }^{5}$ They also appear to require greater stretching force initially, possibly to break up adhesions or intermolecular cross-linkages. ${ }^{6}$ As long as the increase in symptoms is temporary (hours to days) and limited (no significant loss of circulatory flow or neurologic function distally), it is essential to continue progressive stretching. Unfortunately, many people give up at this point, or continue to stretch only one or two times daily, which is usually just enough to continue irritating the neurovascular structures but not enough to create and maintain true elongation of the muscle.

Effective therapeutic stretching, at least initially, usually requires five to ten repetitions per 24 hours. This may sound excessive to patients; however, with proper explanation of the pathophysiologic basis, many will comply. Each stretch session need last only a few minutes, so that even ten times daily may total only 30 minutes in a 24 -hour day. The key is to be persistent, with frequent sessions and attempts to stretch slightly further each time, on the basis of the concept of progressive stretch. There should be some discomfort, often somewhat intense locally and with extremity symptoms as well. However, symptoms should subside within seconds or minutes following the stretch. This has been noted by Kottke ${ }^{6}$ : "Stretching should be past the point of pain, but there should be no residual pain when stretching is discontinued." Swezey ${ }^{8}$ used other criteria: "Exercise. . should not cause significant pain, and any pain brought on during an exercise should subside within 2 hours and no increase of pain attributable to exercise should be present on the day following." Nonetheless, some authors ${ }^{6}$ note that pain could persist up to 24 hours.

The necessity for high-frequency stretching is inherent in the pathophysiologic basis of the syndrome and, for that matter, in most all forms of myofascial pain syndrome. The process of foreshortening or relative contracture of various muscles, with or without development of trigger points, results over a period of weeks, months, or occasionally years. Pain is usually involved, and helps establish a feedback loop with decreased movement and guarding, and then decreased range-of-motion, which results in further shortening. By the time the patient comes to the physician, what could be called an abnormal muscle engram (Fig 5) usually is present. The neural input from the muscle spindle to the cord and brain has been reestablished at a new set level to allow or accommodate the new foreshortened length. The process of shortening, extending 


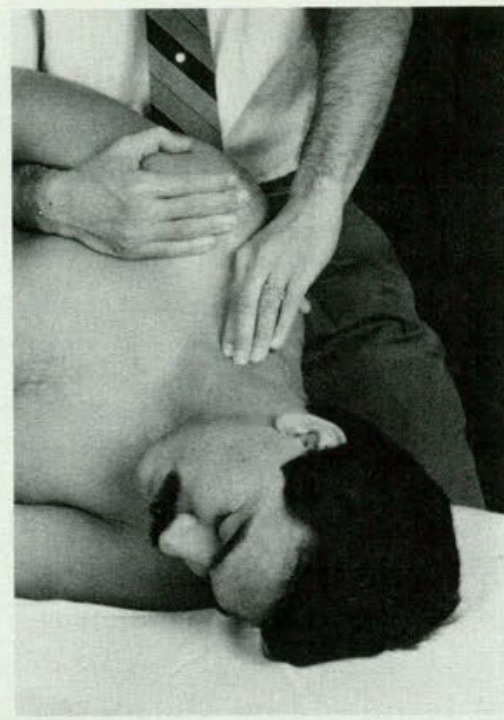

Figure 3. Myofascial release technique for the scalene muscles. Sidelying approach (left). Supine approach with pillow under thorax (right, top). Supine approach with head extended off the table and supported by the operator's knees (right, bottom).
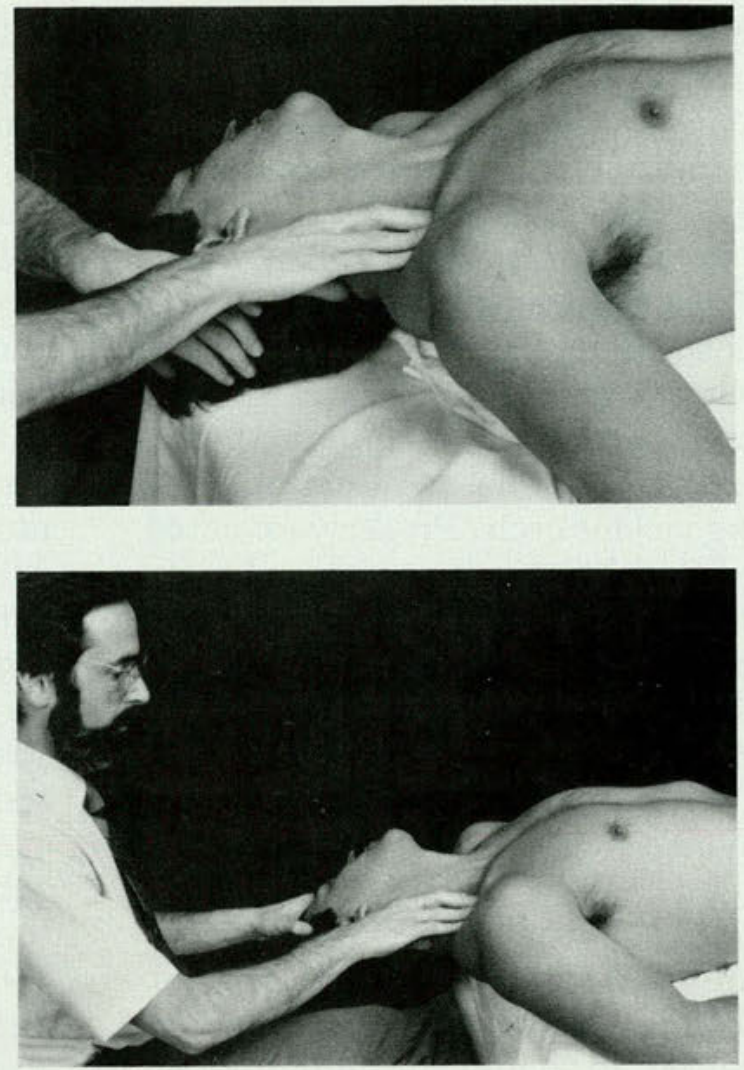

from phases 1 through 3 , (Fig 5) probably develops gradually through a continuum. The separation into phases is only for the purposes of illustration regarding the complexity of neural involvement, which helps to establish and maintain the contracture.

To reverse this process, it is necessary to override the present programming and reprogram the cerebral-spinal patterns, or engram, to accept a new length of the muscle. For most situations, especially chronic cases, it appears that one to two stretches per day is not adequate for reprogramming, probably for two reasons.

First, it is difficult for patients to stretch far enough to lengthen a muscle adequately, at least during any one session, because of pain. Also, it takes time for the muscle and other soft tissues to respond to the tension of a vigorous pull during a stretch. Joynt ${ }^{6}$ noted that connective tissue elongates slowly by the process of "plastic deformation or creep." The muscle will usually begin to "recoil" rapidly in an attempt to withdraw back to the resting length. Kottke ${ }^{7}$ advocated: "Stretching should be repeated in less time than is required for connective tissue to 'set' in a shortened position." This is consistent with comments by Swezey, ${ }^{8}$ who recommended stretching for contractures with a frequency of up to hourly, which involved five to ten stretch repetitions of several seconds' duration. He noted that a prolonged or "static" stretch was preferred. Therefore, it appears that frequent attempts within a relatively short period ( 24 hours) can help overcome this withdrawal or recoil effect by successively reapplying tension before the muscle has returned to its shortened state. Thus, the tendency for the muscle to withdraw is repetitively interrupted, and each time the next stretch session begins with a slightly longer muscle that can elongate further still. Significant shortening will occur overnight; however, the next morning length should be longer than it was 24 hours earlier.

Second, the engram represents a form of programming that has been present for some time, and is therefore not easily amenable to 


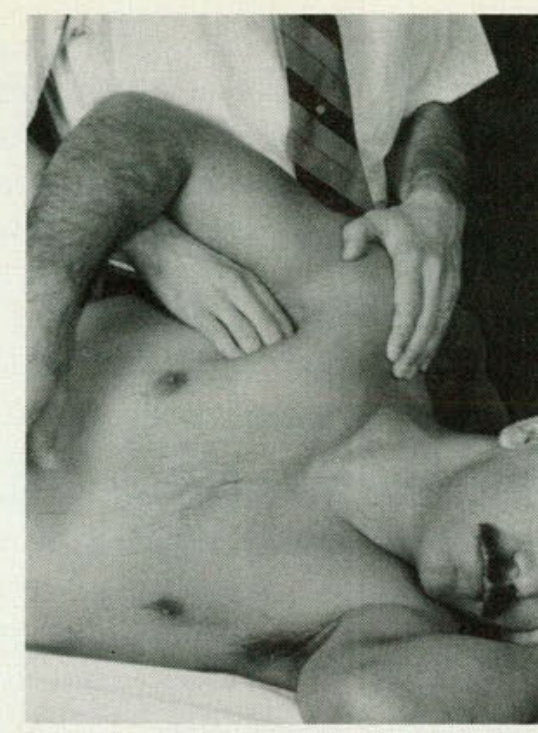

Figure 4. Myofascial release technique for the smaller pectoral muscle. Side-lying approach (left). Supine approach with pillow under the thorax and traction to the arm (right, top). Supine approach with passive scapular retraction (right, bottom).
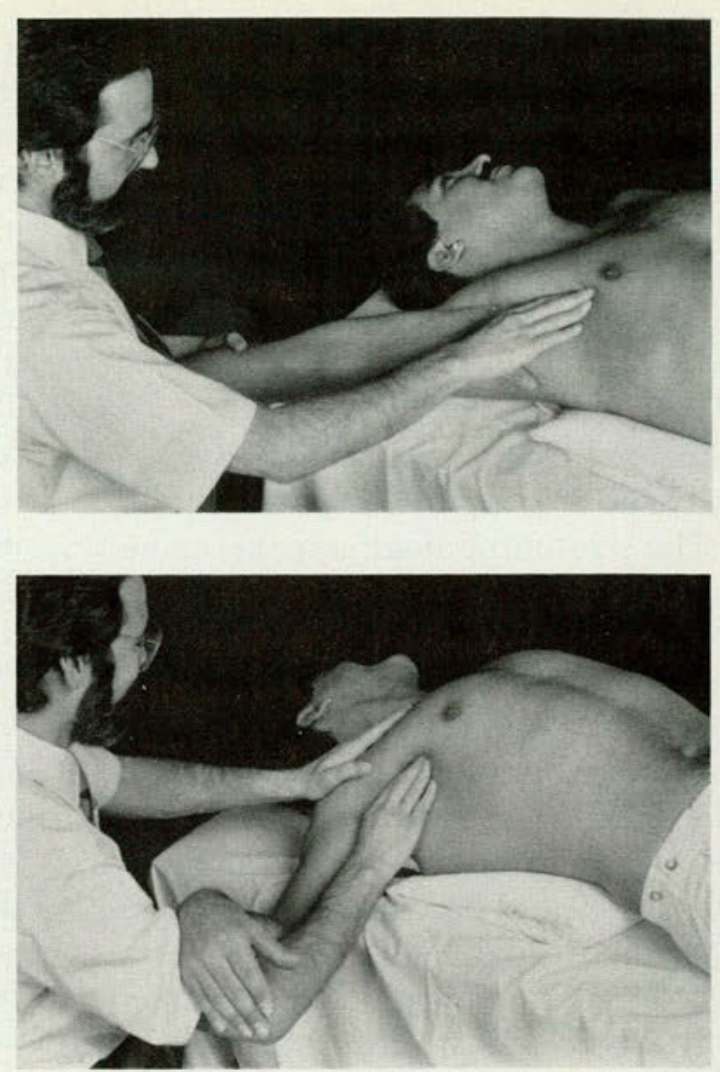

change. The input of one or two mild or gentle stretches, especially if done without pain, does not appear strong enough to override a solid engram. It took several painful seconds, minutes, or days to create the program and it will not usually let go easily or painlessly. Frequent attempts at reprogramming, however, will literally bombard the old program with new input. New input of a longer muscle becomes progressively harder to refuse or block, so the new length eventually becomes accepted.

As part of the process of changing the old painful pattern, this new pain should be seen as a natural consequence. Just as the initial pain of injury or pathologic alteration played a role in limiting movement and function to create a short muscle and its engrams, so, too, does this "healing pain" play its role in reversing the situation. In fact, the discomfort of stretch should be thought of as, and used to, advantage, because it strengthens the input of a longer muscle, and thus reinforces or "saves" the program. Since a longer muscle is one that does not easily allow trigger points to develop or activate, less pain should result.

Another effect that decreases pain may be hyperstimulation analgesia, with the disruption of abnormal reverberating neural circuits by intense stimulation, a theory proposed by Melzack. ${ }^{9} \mathrm{He}$ noted that brief intense stimulation could alter neural pathways facilitated by the pathologic processes in trigger points and related to "memories" of prior injury.

As long as a "ripping, burning, stinging" type of pain is avoided, patients can usually understand and accept the deep, aching pain and "discomfort" that occurs with stretching. Joynt ${ }^{6}$ stated: "The therapist must also be adept at convincing the patient to tolerate a certain degree of discomfort, since discomfort may be necessary to achieve progress." The use of patient education, encouragement, and reinforcement to assist with stretching is supported by Clark and Hunt ${ }^{10}$ :

Goals, purposes and general activity levels also appear to modulate the pain experience....The general, intense sensory input 
can distract the subject, and coding of the input lends cognitive significance to the situation to modify "central processing," and thus the experience. . . exercise, if persistent, in the face of pain, should eventually dampen the pain experience itself. ... the patient may have to be effectively encouraged to attempt and maintain initially painful exercises that are therapeutically important.

This is consistent with what is observed after progressive stretching by the patient independently. The stretching itself appears to become less painful over time.

The need to stretch with great frequency tapers rapidly as the muscle lengthens. In many cases, 2 to 4 days is enough, after which, frequency can be gradually decreased to 1 to 2 times daily as a maintenance level. Each situation will vary in accordance with how "tight" and short the muscle has become and the chronicity of the problem. Joynt ${ }^{6}$ noted: "In soft tissue contractures of long duration, the collagen is usually relatively mature and unlikely to respond rapidly or significantly to stretching."

If patients are not stretching frequently enough, they will have recurrence of pain throughout the day, which should warn them that they should be stretching. Pain recurs because as the muscle shortens beyond a critical point, the trigger points within the muscle are reactivated.

\section{Pressure effects of myofascial release}

Some areas of the body are difficult to stretch adequately. The scalene muscles are not always easy to stretch, especially if any significant degenerative joint disease is present in the cervical spine. Similarly, degenerative changes in the shoulder joint could limit stretch of the pectoral muscles. The smaller pectoral muscle is difficult to stretch in any event, except perhaps if the patient hangs by one hand from a bar, which is not possible for many people for several reasons. Therefore, the technique of localized, manual release (with stretch) is often necessary to release and lengthen the muscle, and may accelerate relief.

This method of treatment should be considered a deep myofascial release technique and a modified form of osteopathic manipulation. It is a powerful soft tissue technique that combines vigorous, controlled stretch through a barrier (or restriction) with direct pressure on the myofascial tissue. As the stretch is increased, the pressure may be intensified and carried along the muscle, parallel to the fibers (and usually in a direction opposite to the stretch). The manual application of pressure is similar to that used with rolfing. However, controlled manipulation of the structure is used simultaneously with precise positioning to facilitate effective stretch during the pressure application. In addition, treatment is directed specifically to a particular muscle and its investing fascia.

Pressure is important because stretching alone, even when performed by the physician (or therapist), is often insufficient to resolve the problem. Pressure applied to soft tissues (as well as bone and cartilage) creates electrical potentials, which in turn can do the following: control connective tissue orientation (and mass), affect the "behavior" of connective tissue cells, or probably even "regulate the cells."11

It has been shown that the effects of electric fields increase collagen synthesis (more than $100 \%$ ) and accelerate healing of soft tissue wounds, and that they probably affect cell nutrition and can control the structure of connective tissue. The basic mechanism involves the conversion of mechanical energy into electrical energy. Apparently this can occur because connective tissue can function as a transducer. ${ }^{11}$ This ability to transform one form of energy into another, and thereby facilitate healing, is consistent with comments made by Little ${ }^{12}$ in a comprehensive review of manipulative management of myofascial syndromes.

Little described the body as a thermodynamic system. He indicated that the total energy of such a system and its surroundings must remain constant, though the energy may be converted from one form to another. He noted that the system could gain or lose energy in two ways: (1) through change in position of the system as a whole in relation to its surroundings, and (2) through changes in internal makeup. He quoted Taylor ${ }^{13}$ : 
A.Phase I: Local

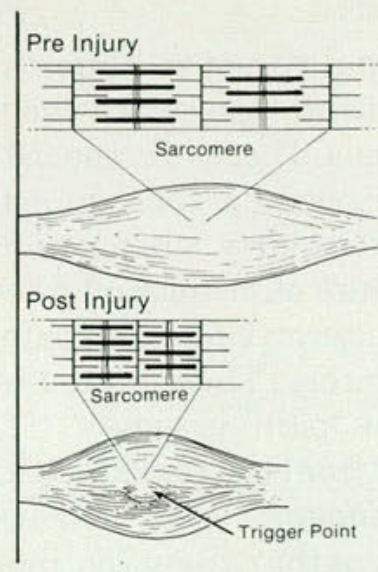

B. Phase II: Reflex

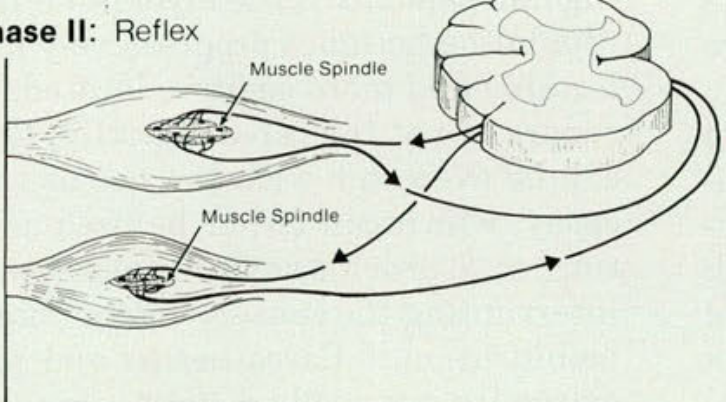

C. Phase III: Central

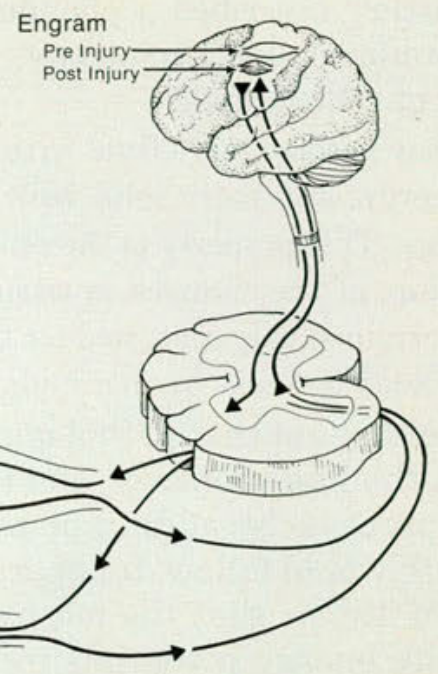

Figure 5. Development of muscle engram. A: Local/muscle. After local trauma, the muscle reactively withdraws, with progressive sarcomere shortening. B: Reflex/spinal. As time progresses, the feedback loop from the muscle to the cord and back becomes more established, with a "spinal engram" perhaps developing for the new length (shortened muscle). C: Central/cerebral-spinal. After weeks or months of ascending input, a well-established higher level engram has developed, which now feeds back to the cord and then the spindle (gamma efferent) to help maintain the shortened muscle.

Whenever poor posture shifts the resultant of force away from the center of mass. . . the downward vector of the earth's pull causes some. . parts of the body's mass to spill over the side of the container, so to speak .... [This] results in abnormal stresses and strains. ... These areas. . .are deprived of the normal amounts of free energy and thereby become areas of stagnation and stasis.

Little ${ }^{12}$ further elaborated: "The areas of particular susceptibility to energy loss, and therefore to stagnation and stasis, are the ground substance and fibro-areolar laminae of the fascia."

This "stagnation" effect could account for the buildup of substances in trigger points, as described by Awad, ${ }^{14}$ which included acid mucopolysaccharides, probably resulting in stiff- ness or "gelling." The mucopolysaccharides attract water and some edema results, creating, in effect, a "space occupying lesion" between the muscle fibers. ${ }^{14}$

Under these circumstances, it would appear that firm, local pressure probably "milks out" or removes the mucopolysaccharide (and other wastes or perhaps even calcium) accumulations, releases or "frees up" focal myofascial adhesions/restrictions, and "guides" the myofascial unit back into more proper alignment and function. The taut band described by Simons ${ }^{15}$ has abnormally short sarcomeres near the trigger point (and abnormally long ones further away). The direct pressure probably helps normalize the sarcomere length within the band, as the stretch alone may not accomplish this effect. 
Little $^{12}$ described a phenomenon of thermodynamics called thixotropy, which is a property of certain gels:

They become more fluid with the addition of energy, and more solid with the loss of energy. This property is the essential basis for many of the changes in tissue-feel detected by trained palpation, and for the modification of such changes by manipulation.

He thought that pathologic processes began with the loss of energy, and that by reversing the process, by adding or restoring energy, health would follow. It apparently was the belief of Taylor that the most effective ways to modify energy potentials in abnormal tissue were through the use of manipulative pressure and stretching.

Little $^{12}$ noted that one of the most effective methods of managing myofascial tissues manipulatively was through the technique of postural release taught by Rolf. Little stated: "This pressure, judiciously and slowly applied in specific directions with awareness of the changes taking place during the process, results in myofascial lengthening and softening, and lessened tension. The tissues become more fluid and less gelled."

It should be obvious at this point that the treatment may cause significant discomfort. The patient usually already has a complaint of pain from local muscular changes as well as secondary compressive effects on neurovascular structures. The addition of self-stretching is uncomfortable, as noted previously, and the myofascial release technique adds local pressure. This is why it is important to explain the mechanisms involved to the patient. Rolf ${ }^{16}$ has said: "Humans resist change. There is no painful experience, apart from the motor intent to withdraw from the experience," which is usually what happens when most patients attempt to begin stretching on their own for TOS. Unfortunately, as Rolf ${ }^{16}$ continues:

The experience of change to the average man often manifests itself as pain. ... They verbalize their resistance as pain, emotional or physical. All too often their emotional pain-their depression, their grief, even their anger-is a perception of physiological imbalance, an awareness of chemical lacks or overloads in blood and tissues.

This emotional response, unfortunately, is something that interferes with the treatment on a regular basis. Therefore, the patient must be educated, especially regarding the use of stretching, particularly when combined with the direct pressure of myofascial release. Rolf ${ }^{16}$ further elaborates on this: "The pain of fascial change is transitory. The minute the pressure is removed, the 'pain' is gone. ...This is entirely different from the residual 'pain' following hurt or damage."

Additionally, as the patient continues stretching independently, the myofascial release manipulation becomes progressively less uncomfortable and more easily tolerated. If the procedure is not tolerated initially, this usually can be overcome with the use of vapocoolant spray, which can either be used as a distraction $^{5}$ or to provide surface anesthesia, thereby interrupting the sensory-motor chain to allow mobilization. ${ }^{17}$ Paresthesias and pain in the extremity, especially distally, appear to resolve first, with proximal pain requiring several additional treatments.

The myofascial release technique can be thought of as having at least three specific actions:

- Release of tissue tension, restriction, or contracture.

- Re-energizing effects on the tissue by "adding" energy from an outside source with direct pressure, and "converting" energy from mechanical to electrical.

- Reprogramming effects on the central nervous system by intensifying the input or programming that occurs as the muscle achieves a new length (and creates a new engram).

This threefold effect perhaps explains the enhanced or rapid response noted in TOS, where the several pathologic mechanisms involved tend to make TOS resistant to other forms of conservative treatments. The speed of development and strength of the engram formation appears to be secondary to the following: (1) severity of injury; (2) degree of pain experienced; (3) secondary gain, anger, 
anxiety, expectations of the patient; and (4) amount of sleep disturbance, if present. The ability to reverse the process and break the cycle is probably dependent on all of these four factors with the addition of the following: the resolve of the physician, his or her ability to educate the patient regarding the process, and the ability of the patient to become an active participant in treatment.

1. Sucher BM: Thoracic outlet syndrome-A myofascial variant: Part 1. Pathology and diagnosis. JAOA 1990;90:686-704. 2. Peet RM, Hendriksen JD, Anderson TP, et al: Thoracic outlet syndromes: Evaluation of a therapeutic exercise program. Proc Mayo Clin 1956;31:281-285.

3. Di Giovanna EL: Osteopathic management of thoracic outlet syndrome. Osteopathic Medical News July-August 1988;5:40-43.

4. Dobrusin R: An osteopathic approach to conservative management of thoracic outlet syndromes. JAOA 1989;89:10461957.

5. Travell JG, Simons DG: Myofascial Pain and Dysfunction: The Trigger Point Manual. Baltimore, Williams \& Wilkins Co, 1983.

6. Joynt RL: Therapeutic exercise, in DeLisa JA: Rehabilitation Medicine: Principles and Practice. Philadelphia, JB Lippincott, 1988, pp 346-371.

7. Kottke FJ: Therapeutic exercise to maintain mobility, in Kot- tke FJ, Stillwell KG, Lehmann JF (eds): Krusen's Handbook of Physical Medicine and Rehabilitation, ed 3. Philadelphia, WB Saunders Co, 1982, pp 389-402.

8. Swezey RL: Arthrosis, in Basmajian JV, Kirby RL (eds): Medical Rehabilitation. Baltimore, Williams \& Wilkins Co, 1984, pp 216-218.

9. Melzack R: Myofascial trigger points: Relation to acupuncture and mechanisms of pain. Arch Phys Med Rehabil 1981;62:114-117.

10. Clark WC, Hunt HF: Pain, in Downey JA, Darling RC (eds): Physiological Basis of Rehabilitation Medicine. Philadelphia, WB Saunders Co, 1971, pp 373-401.

11. Bassett CAL: Effect of force on skeletal tissues, in Downey JA, Darling RC (eds): Physiological Basis of Rehabilitation Medicine. Philadelphia, WB Saunders Co, 1971, pp 283-316.

12. Little KE: Toward more effective manipulative management of chronic myofascial strain and stress syndromes. JAOA 1969;68:675-685.

13. Taylor R: Bioenergetics of man. Academy of Applied Osteopathy Year Book, 1958, pp 91-96, cited in Little KE: Toward more effective manipulative management of chronic myofascial strain and stress syndromes. JAOA 1969;68:675-685.

14. Awad EA: Interstitial myofibrositis: Hypothesis of the mechanism. Arch Phys Med Rehabil 1973;54:449-453.

15. Simons DG: Myofascial pain syndrome due to trigger points, in Goodgold J: Rehabilitation Medicine. St. Louis, CV Mosby Co, 1988, pp 686-723.

16. Rolf IP: Rolfing: The Integration of Human Structures. New York, Harper \& Row, 1977, pp 275-283.

17. Kraus H (ed): Diagnosis and Treatment of Muscle Pain. Chicago, Quintessence Publishing Co, 1988, pp 11-20. 